ДИФФЕРЕНЦИАЛЬНЫЕ УРАВНЕНИЯ, ДИНАМИЧЕСКИЕ СИСТЕМЫ И ОПТИМАЛЬНОЕ УПРАВЛЕНИЕ

Маторин С.И.*, «Белгородский университет кооперации,

Белов С.П., Жихарев А.Г. экономики и права»,

г. Белгород, Россия,

*matorin@bsu.edu.ru

\section{УЧЕТ ОБЩЕСИСТЕМНЫХ ЗАКОНОМЕРНОСТЕЙ ПРИ МОДЕЛИРОВАНИИ СЛОЖНЫХ СИСТЕМ СРЕДСТВАМИ СИСТЕМНО-ОБЪЕКТНОГО ПОДХОДА}

Введение:

актуальность исследования обусловлена отсутствием обоснования общесистемных законов и принципов с единых позиций в рамках традиционной теоретико-множественной теории систем, на основе которой построены существующие методологии анализа и синтеза систем.

Методы

исследования:

результаты, полученные в рамках настоящей статьи, являются продолжением научно-исследовательской работы по созданию формализованной теории имитационного моделирования процессов и систем. Ранее авторами были разработаны формальные основы исчисления функциональных объектов с применением исчисления объектов Абади-Кардели. Были введены такие термы, как: потоковый и узловой объекты. Определены формальные основы состояний потоковых и узловых объектов, а также их взаимосвязи. Помимо реализации процедур синтеза и анализа систем, полученные результаты открыли перспективы фрормализованного учета в системно-объектных моделях общесистемных принципов и закономерностей. В работе с использованием вышеупомянутого исчисления объектов Абади-Кардели уточняется определение системы в рамках системно-объектного подхода «Узел-Функция-Объект», а также предложена формализация понятий «системообразующий фактор» и «адаптация системы».

Результаты

исследования и их обсуждение:

представленные формализмы использованы для учета общесистемных закономерностей при построении графоаналитических 
моделей организационно-деловых и производственно-технологических процессов в терминах «Узел-Функция-Объект». Описанный подход позволяет распределить известные общесистемные закономерности на три группы: связанные со структурными свойствами систем, связанные с их фуннцциональными свойствами и с субстанциальными свойствами, что обосновывает иерархическую зависимость между ними. Формально показано, что принцип организационной непрерывности выполняется только при соблюдении принципа моноцентризма, понимаемого расширенно. Исследованы возможности системно-объектного, системно-структурного, объектно-ориентированного подходов и нотации BPMN учитывать общесистемные закономерности в графоаналитических моделях.

авторами сделан вывод, что системно-объектный подход «Узел-Функция-Объект», позволяет учитывать с единых позиций существующие общесистемные законы и принципы в большей степени, чем другие подходы. Что, в свою очередь, открывает новые перспективы имитационного моделирования процессов и систем, а именно: процессов адаптации и эволюции систем. Ключевые слова: системный подход «Узел-Функция-Объект», общесистемные закономерности, графооаналитическое моделирование.

\footnotetext{
Matorin S.I.*,

Belov S.P.,

«Belgorod University of cooperation,

Zhikharev A.G.

Economics and law»,

Belgorod, Russia,

*matorin@bsu.edu.ru
}

\section{ACCOUNTING FOR SYSTEM-WIDE REGULARITIES IN THE MODELING OF COMPLEX SYSTEMS BY MEANS OF THE SYSTEM-OBJECT APPROACH}

Introduction:

the relevance of the study is due to the lack of justification of system-wide laws and principles from a unified position within the framework of the traditional set theory of systems, on the basis of which existing methodologies of analysis and synthesis of systems are constructed.

Research methods: the results obtained within the framework of this article are a continuation of the research work on the creation of a formalized theory of simulation modeling of processes and systems. Earlier, the authors developed formal bases for the calculation of functional objects using the Abad-Kardeli calculus. There were introduced such terms as: stream and node objects. The formal foundations of the states of flow and core objects, as well as their interrelations, are determined. In addition to implementing the procedures for synthesis and analysis of systems, the results obtained opened the prospects for formalized accounting in system-object models of system-wide principles and regularities. In work with the above-mentioned calculation of Abadi-Kardeli objects, the definition of the system within the system-object approach "Node-Function-Object" is clarified, and a formalization of the concepts "system-forming factor" and "system adaptation" is proposed. 
Results of the study

and their discussion: the presented formalisms were used to take into account system-wide regularities in the construction of graphoanalytical models of organizational-business and industrial-technological processes in terms of "Node-Function-Object". The described approach makes it possible to distribute known system-wide regularities into three groups: related to the structural properties of systems related to their functional properties and to substantial properties, which justifies the hierarchical dependence between them. Formally, it is shown that the principle of organizational continuity is fulfilled only if the principle of monocentrism is observed, understood broadly. The possibilities of system-object, system-structural, object-oriented approaches and BPMN notation to consider system-wide regularities in graphoanalytical models are investigated.

Conclusions: $\quad$ the authors concluded that the system-object approach "Node-Function-Object" allows to take into account from one position the existing system-wide laws and principles to a greater extent than other approaches. That, in turn, opens up new prospects for simulation of processes and systems, namely: the processes of adaptation and evolution of systems.

Key words: $\quad$ system approach "Node-Function-Object", general-regular regularities, graphoanalytical modeling.

\section{Введение}

В настоящее время при моделировании сложных систем (организационно-деловых и производственно-технологических процессов) широко применяются различные графические языки, например, средства системно-структурного или системно-функционального анализа DFD, IDEF0, IDEF3; средство объектного анализа UML; нотация BPMN (гибрид UML и IDEF3). Хотя эти средства/языки часто позиционируются как средства системного анализа, в их документации и примерах их использования не отмечаются возможности и последствия учета, так называемых, общесистемных закономерностей [1], большинство из которых были установлены во времена бурного развития системных исследований. В рамках традиционной теоретико-множественной теории систем эти закономерности только констатируются и описываются, но не обосновываются с единой позиции концептуальными средствами системного подхода ни по отдельности, ни во взаимодействии между собой [2]. Эти закономерности и их взаимосвязи получили обоснование в рамках системно-объектного подхода «Узел-Функция-Объект» (УФО-подход) [3, 4].

\section{Метод исследования}

B рамках УФО-подхода система s представляется в виде специального объекта исчисления объектов Абади-Кардели [5, 6] (называемого нами «узловым объектом»), состоящего из полей и методов:

$$
\mathbf{s}=\text { [us, fs, Os] }
$$

где: us - $\quad$ узел, формально представляет собой поле узлового объекта для описания объектов еще одного специального вида (называемых нами «потоковыми объектами»), соответствую- 
щих множеству функциональных связей данной системы us $\leftrightarrow$ Ls? $\smile$ Ls!, где Ls? - множество входящих интерфейсных потоковых объектов, соответствующих входящим связям системы s, Ls! - множество исходящих интерфейсных потоковых объектов, соответствующих выходящим связям системы s. Причем: Ls? $\smile \mathbf{L}$ и $\mathbf{L} \mathbf{s} ! \subset \mathbf{L}$, т. е. относятся к множеству всех связей $\mathbf{L}$ (потоковых объектов).

fs - $\quad$ функция, формально представляет собою метод узлового объекта, описывающий функцию системы s, т.е. процесс преобразования входящих интерфейсных потоковых объектов (входящих связей системы) Ls? в выходящие Ls!. Далее, в соответствии с принятой в теории объектов манерой обозначений, метод узлового объекта будем представлять в следующем виде: fs(Ls?)Ls!, где fs - метод узлового объекта (функция/процесс системы s) с областью определения Ls? и областью значений Ls!, соответственно;

Os - объект, формально представляющий собою множество полей узлового объекта для описания объектных (субстанциальных) характеристик системы s. Множество полей для описания объектных характеристик системы состоит из трех подмножеств: Os = Os? ๖ Os!, где Os? - множество полей, которое содержит интерфейсные входные характеристики узлового объекта (системы s), Os! - множество полей, которое содержит интерфейсные выходные характеристики узлового объекта (системы s), Osf - множество полей, которое содержит передаточные характеристики узлового объекта (системы s).

Таким образом, в полном соответствии с содержательным определением системы как функционального объекта, функция которого обусловлена функцией объекта более высокого яруса (т.е. надсистемы) [7], система s, формально представляется в виде следующего выражения:

$$
\text { s = [(Ls?, Ls!); fs(Ls?)Ls!; (Os?, Os!, Osf)]. }
$$

Упомянутое в определении системы явление обуславливания функции системы функцией надсистемы рассматривается как функциональный запрос надсистемы на систему с определенной функцией (внешняя детерминанта системы). Эта детерминанта формально описывается полем us узлового объекта, соответствующего данной системе, т.е. множеством потоковых объектов, соответствующих функциональным связям системы (Ls?, Ls!). Внешняя детерминанта системы есть причина ее возникновения, цель ее существования и главный определитель ее структурных, функциональных 
и субстанциальных свойств. Таким образом, она рассматривается в качестве универсального системообразующего фактора. Функционирование же системы под влиянием внешней детерминанты является ее внутренней детерминантой, так как непосредственно определяет ее внутренние свойства (структурные, функциональные и субстанциальные свойства подсистем). Эта детерминанта формально описывается методом fs узлового объекта, соответствующего данной системе, т. е. в следующем виде: fs(Ls?)Ls!. Кроме того, функционирование системы в соответствии с внешней детерминантой устанавливает между системой и надсистемой отношение поддержания функциональной способности более целого. Таким образом, можно уточнить формальное выражение для принятого определения системы следующим образом:

$$
\mathbf{s}=[\mathrm{us} \Rightarrow \mathbf{f s} \Rightarrow \mathbf{O s}] \text { и } \Rightarrow[(L s ?, \text { Ls!) } \Rightarrow \mathbf{f s}(L s ?) L s ! \Rightarrow(\text { Os?, Os!, Osf })]
$$

Процесс приближения внутренней детерминанты (fs(Ls?)Ls!) системы к ее внешней детерминанте (Ls? $\smile \mathbf{L s} ! \leftrightarrow \mathbf{u s})$ представляет собой адаптацию системы к запросу надсистемы [7]. Полностью адаптированной считается система, у которой внутренняя детерминанта полностью соответствует внешней, т. е. fs $\leftrightarrow$ us. Такая система функционирует в полном соответствии с функциональным запросом надсистемы. Сама адаптация представляет собой процесс приближения текущего функционирования системы к требуемому, т.e. $\mathbf{f s}_{\text {тек }} \rightarrow \mathbf{f s} \leftrightarrow \mathbf{u s}$. Узел us определяет область определения и область значений функции (Dfs = Ls? и Efs = Ls!) системы s. Параметры преобразования/ отображения fs:Ls? $\rightarrow$ Ls! определяются объектными характеристиками Os системы s, задающими конкретные параметры приема системой входных потоков (Os?), параметры выполнения процесса fs (Osf) и параметры выдачи системой выходных потоков (Os!). Таким образом, приближение $\mathbf{f s}_{\text {тек }}$ к fs обусловлено соответствующим изменением Os $=\mathbf{O s ?} \smile$ Os! $\smile$ Osf.

Для анализа возможностей системно-объектного подхода учитывать общесистемные закономерности необходимо эти закономерности описать, используя приведенные выше понятия.

\section{Результаты исследования и их обсуждение}

\section{Структурные (узловые) закономерности систем.}

Принцип коммуникативности (система связана множеством коммуникаций с окружающей средой). В процессе графоаналитического моделирования средствами УФО-подхода данный принцип учитывается, в первую очередь, на контекстной диаграмме в виде функциональных (внешних) связей системы, которые далее замыкаются через внутренние (поддерживающие) связи данной системы на диаграммах декомпозиции разного уровня. Системнообъектный подход требует обязательного наличия хотя бы одного входа и, хотя бы одного выхода у любой системы на любом уровне иерархии. 
Системно-структурные (системно-функциональные) средства графоаналитического моделирования также обеспечивают учет принципа коммуникативности на контекстной диаграмме. Однако, для диаграмм декомпозиции существуют оговорки. Например, системы, представляемые своими процессами, в IDEF0 и IDEF3 могут не иметь входных связей [8]. Объектно-ориентированные средства моделирования учитывают данный принцип с точки зрения контекста в некоторой степени только на диаграмме вариантов использования в виде связей с акторами. При этом не все из них являются потоковыми объектами и нет обязательного требования наличия и входа, и выхода [9]. Нотация BPMN вообще не предусматривает контекстного уровня описания системы и представляемые на BPD диаграммах связи так же, как и в языке UML, могут не представлять входных и выходных потоков моделируемых систем [10].

Принцип иерархичности (система на любом ярусе иерархии является частью системы более высокого яруса, т.е. надсистемы). Данный принцип работоспособен только при выполнении принципа коммуникативности, что хорошо согласуется с содержательной трактовкой этих принципов в терминах системно-объектного подхода [3]. В процессе графоаналитического моделирования средствами УФО-подхода $[11,12]$ данный принцип учитывается в виде иерархии диаграмм декомпозиции произвольного числа уровней.

Системно-структурные средства графоаналитического моделирования также обеспечивают учет принципа иерархичности в виде иерархии диаграмм. Объектно-ориентированные средства моделирования учитывают данный принцип только для так называемых внешних систем (систем-классов) в виде диаграммы классов. Для систем-явлений (объектов) данный принцип не учитывается. Нотация BPMN предусматривает только три уровня иерархии, т.е. не учитывает данный принцип в полном объеме.

Принцип моноцентризма (по Богданову [13]: устойчивая система обладает одним центром). С точки зрения авторов, трактующих данный принцип более широко [14], принцип моноцентризма работоспособен только при выполнении принципа иерархичности, что также хорошо согласуется с содержательной трактовкой этих принципов. В процессе графоаналитического моделирования средствами УФО-подхода данный принцип учитывается в виде контекстной диаграммы с одной системой.

Системно-структурные средства графоаналитического моделирования также обеспечивают учет принципа моноцентризма в виде контекстной диаграммы с одним процессом. Объектно-ориентированные средства моделирования, как и нотация BPMN данный принцип не учитывают.

Принцип организационной непрерывности (констатирует факт наличия между всякими двумя системами звеньев, вводящих их в одну «цепь ингрессии»). Используя упомянутые выше термины и обозначения данный принцип можно представить в виде следующего выражения: $\forall\left(\mathbf{u s}_{\mathbf{i}} \wedge \mathbf{u s}_{\mathbf{j}}\right) \mathbf{g L}_{\mathrm{ij}}: \mathbf{L}_{\mathbf{i j}}$ э $\left(\mathbf{L} \mathbf{s}_{\mathbf{i}} \wedge \mathbf{L} \mathbf{s}_{\mathbf{j}}\right)$, где $\mathbf{u s}_{\mathbf{i}}-$ узел системы $\mathbf{s}_{\mathbf{i}} ; \mathbf{u s}_{\mathbf{j}}-$ узел системы $\mathbf{s}_{\mathbf{j}} ; \mathbf{L}_{\mathbf{i j}}-$ множество (цепочка) связей между $\mathbf{s}_{\mathbf{i}}$ и $\mathbf{s}_{\mathbf{j}} ; \mathbf{L s}_{\mathbf{i}}-$ множество связей $\mathbf{s}_{\mathbf{i}} ; \mathbf{L s}_{\mathbf{j}}-$ множество связей $\mathbf{s}_{\mathrm{j}}$. Можно показать, что данный принцип, также установленный Богдановым [13], выполняется только в условиях моноцентризма, понимаемого рас-

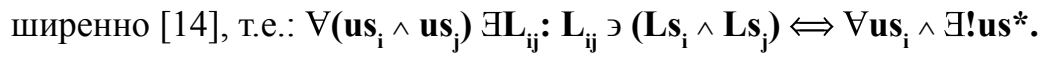


Пусть система $\mathbf{s}_{\mathbf{i}}$ является частью иерархии систем так, что: $\mathbf{u s} \mathbf{s}_{\mathbf{i}} \in \mathbf{u s}{ }^{1 \mathbf{i}} \in$ $\mathbf{u s}^{2 \mathbf{i}} \in \mathbf{u} \mathbf{s}^{3 \mathrm{i}} \in \ldots \in \mathbf{u} \mathbf{s}^{\mathrm{n}}$, т.е. самый верхний уровень данной иерархии представляет собой систему $\mathbf{s}^{\mathrm{n}}$. Система $\mathbf{s}_{\mathbf{j}}$ является частью иерархии систем так, что: $\mathbf{u s}_{\mathbf{j}}$ $\in \mathbf{u s}^{1 \mathbf{j}} \mathbf{u s}^{2 \mathbf{j}} \in \mathbf{u s}^{\mathbf{3 i}} \in \ldots \in \mathbf{u} \mathbf{s}^{\mathbf{m}}$, т.е. самый верхний уровень данной иерархии представляет собой систему $\mathbf{s}^{\mathbf{m}}$. Допустим, что $\forall \mathbf{u s}$ 马! us*: us* $\ni$ us не верно (принцип моноцентризма не выполняется), т.е. $\mathbf{u s}^{\mathbf{n}} \notin \mathbf{u s}^{*}$ и $\mathbf{u s}^{\mathbf{m}} \notin \mathbf{u s}^{*}$. Тогда $\forall\left(\mathbf{u s}_{\mathbf{i}}\right.$ $\left.\wedge \mathbf{u s}_{\mathbf{j}}\right): \mathbf{L} \mathbf{s}_{\mathbf{i}} \cap \mathbf{L} \mathbf{s}_{\mathbf{j}}=\varnothing$ Следовательно, $\mathbf{L}_{\mathbf{i j}}$ не является множеством связей между всякими двумя системами, т.е. принцип организационной непрерывности не выполняется. Полученный вывод можно рассматривать как дополнительный аргумент в пользу расширенного понимания моноцентризма.

В процессе графоаналитического моделирования средствами УФОподхода данный принцип учитывается путем прослеживания связей между системами в рамках всей модели.

Системно-структурные средства графоаналитического моделирования также обеспечивают учет данного принципа путем прослеживания в модели связей между процессами. Объектно-ориентированные средства моделирования позволяют прослеживать связи только в рамках одного прецедента, так как прецеденты зачастую не связаны между собой. Нотация BPMN обеспечивает учет данного принципа путем прослеживания связей в рамках одной BPD-диаграммы.

Принцип обратной связи (устойчивость в сложных динамических системах достигается за счёт замыкания петель обратных связей). Данный принцип работоспособен только при выполнении принципа коммуникативности, что также хорошо согласуется с содержательной трактовкой этих принципов. В процессе графоаналитического моделирования средствами УФО-подхода данный принцип учитывается в виде двухсторонних связей и на контекстной диаграмме, и на диаграммах декомпозиции в соответствии с правилом замкнутости, входящим в концепцию системной теории, основанной на системно-объектном подходе [4].

Системно-структурные средства графоаналитического моделирования и нотация BPMN также обеспечивают учет данного принципа в виде двухсторонних связей. Объектно-ориентированные средства моделирования данный принцип не учитывают.

Принцип внешнего дополнения (восходящие к системному центру воздействия координируемых элементов подвергаются своеобразному «обобщению», а нисходящие от системного центра координационные импульсы подвергаются «специфицированию» в зависимости от характера локальных процессов за счет обратных связей от этих процессов). Данный принцип работоспособен только при выполнении принципа иерархичности с учетом принципа обратной связи. В процессе графоаналитического моделирования средствами УФО-подхода данный принцип учитывается возможностью содержательной декомпозиции материальных и информационных потоков при декомпозиции 
процессов и объектов, поскольку в концепцию системно-объектного подхода входит содержательная категориальная классификация потоков связей [14].

Системно-структурные средства графоаналитического моделирования также обеспечивают учет данного принципа в виде возможности содержательной декомпозиции материальных и информационных потоков, но содержательная классификация потоков не определена. Объектно-ориентированные средства моделирования и нотация BPMN данный принцип не учитывают, так как не рассматривают связи как потоки объектов.

Принцип взаимно-дополнительных соотношений/комплиментарности (устойчивость системы достигается взаимно-дополнительными связями между её элементами в виде замкнутых контуров обратных связей). Данный принцип работоспособен только при выполнении принципа обратной связи. По сути дела, это один и тот же принцип. По мнению авторов, принцип обратной связи следует рассматривать как потенциальную возможность существования обратных связей, а принцип комплиментарности как реализацию (экстенцию) такой возможности для обеспечения устойчивости системы. В процессе графоаналитического моделирования средствами УФО-подхода данный принцип учитывается, как принцип обратной связи, в виде двухсторонних связей и на контекстной диаграмме, и на диаграммах декомпозиции в соответствии с правилом замкнутости, входящим в концепцию системной теории, основанной на системно-объектном подходе [4].

Системно-структурные средства графоаналитического моделирования и нотация BPMN также обеспечивают учет данного принципа в виде двухсторонних связей. Объектно-ориентированные средства моделирования данный принцип не учитывают.

\section{Функциональные закономерности систем.}

Гипотеза семиотической непрерывности (система есть образ её среды, т.е. система как элемент окружающей среды отражает некоторые существенные ее свойства). Согласно системно-объектного подхода главной составляющей среды, окружающей систему, является ее надсистема. Эта надсистема «отображает» свою функциональность на функциональность системы с помощью функционального запроса на систему с определенной функцией (внешней детерминанты) и, таким образом, система своим функционированием (внутренней детерминантой) «отражает» некоторые функциональные (т.е. самые существенные) свойства своей надсистемы. При этом функциональный запрос надсистемы (внешняя детерминанта системы) представляет собой набор связей запрашиваемой системы с другими системами, т.е. узел данной системы. Данное представление семиотической непрерывности позволяет рассматривать данную закономерность не как гипотезу, а как основу всех общесистемных закономерностей, связанных с функциональными характеристиками систем. Очевидно, что гипотеза семиотической непрерывности справедлива только при выполнении принципа иерархичности. В процессе графоаналитического моделирования средствами УФО-подхода данный принцип учитывается таким образом, что функциональный запрос надсистемы к системе (т.е. самое существенное ее свойство) отображается в виде требуемых функциональных связей системы, т.е. в виде узла, на любом уровне иерархии. 
Системно-структурные средства графоаналитического моделирования также обеспечивают учет данного принципа в виде требуемых функциональных связей процессов на различных уровнях иерархии, представляющих собой результат функционирования процесса на вышестоящем уровне. Объектно-ориентированные средства моделирования данный принцип могут учесть только на единственной диаграмме вариантов использования в виде связей с акторами. Нотация BPMN данные принцип не учитывает.

Принцип прогрессирующей механизации (части системы в ходе ее развития специализируются или становятся фиксированными по отношению к определенным функциям или механизмам). Данный принцип работоспособен только при условии справедливости гипотезы семиотической непрерывности. В процессе графоаналитического моделирования средствами УФОподхода данный принцип учитывается таким образом, что подсистемы на диаграммах декомпозиции любого уровня возникают как следствия существования граничных связей, т.е. связей верхнего уровня иерархии.

Системно-структурные средства графоаналитического моделирования также обеспечивают учет данного принципа таким образом, что подпроцессы на различных уровнях иерархии возникают как следствия существования граничных связей вышестоящего уровня. Объектно-ориентированные средства моделирования данный принцип не учитывают. Нотация BPMN может учесть данный принцип только до третьего уровня вложенности.

Принцип актуализации функций (объект выступает как организованный лишь в том случае, если свойства его частей (элементов) проявляются как функции сохранения и развития этого объекта), работающий аналогично принципу самоорганизации (процесс поступательной функционализации элементов системы). Данный принцип и принцип прогрессирующей механизации описывают одно и тоже явление функционального соответствия элементов системы разного уровня иерархии, но с разных сторон: прогрессирующая механизация - с верху в низ, актуализация функций - с низу в верх. Соответственно данный принцип работает только при выполнении предыдущего, так как существование любой системы обусловлено функциональным запросом надсистемы на систему с определенной функцией. В процессе графоаналитического моделирования средствами УФО-подхода данный принцип учитывается таким образом, что подсистемы на иерархии диаграмм любого уровня декомпозиции являются поддерживающими частями систем верхнего уровня.

Системно-структурные средства графоаналитического моделирования также обеспечивают учет данного принципа таким образом, что подпроцессы на различных уровнях иерархии поддерживают функционирование процесса на вышестоящем уровне. Объектно-ориентированные средства моделирования данный принцип не учитывают. Нотация BPMN может учесть данный принцип только до третьего уровня вложенности. 
Закон иерархических компенсаций (в системе рост разнообразия на верхнем уровне иерархии обеспечивается его ограничением на более низких уровнях) и закон необходимого разнообразия (для создания системы, способной справится с решением проблемы, обладающей определенным разнообразием, необходимо обеспечить, чтобы система имела большее разнообразие возможностей, чем разнообразие решаемой проблемы). Данные законы работоспособны только при условии выполнения принципа прогрессирующей механизации. Эти две закономерности соотносятся друг с другом также как принципы прогрессирующей механизации и актуализации функций. В процессе графоаналитического моделирования средствами УФОподхода последние два закона учитываются таким образом, что в модели системы процессы верхнего уровня соответствуют более общим разнообразным функциям. Процессы нижнего уровня соответствуют более специальным ограниченным функциям.

Таким же образом учитывают данные законы системно-структурные средства графоаналитического моделирования и нотация BPMN. Объектноориентированные средства моделирования данный принцип не учитывают.

\section{Субстанциальными (объектные) закономерности систем.}

Принцип совместимости (условием взаимодействия между системами является наличие у них относительной совместимости, то есть относительной качественной и организационной однородности). Данный принцип работоспособен только при условии выполнения принципа организационной и гипотезы семиотической непрерывности. В процессе графоаналитического моделирования средствами УФО-подхода данный принцип учитывается установленными правилами системной композиции, базовой иерархией связей и классификацией узлов [3].

Принцип эквифинальности (способность системы достигать состояния, которое не зависит от времени и начальных условий, а зависит только от параметров системы). При этом узловая характеристика системы us pacсматривается как ее важнейший параметр, т.к. представляет собой функциональный запрос надсистемы, определяющий причину и смысл существования данной системы. Данный принцип работоспособен только при условии выполнения принципа самоорганизации, т.е. «эквифинальное» состояние достигается за счет поступательной функционализации элементов системы, направляемой ее внешней детерминантой us. B процессе графоаналитического моделирования средствами УФО-подхода данный принцип учитывается в виде диаграмм декомпозиции, состояние которых зависит только от контекстных диаграмм, отображающих функциональный запрос на систему с определенной функцией. Закон минимума (устойчивость системы определяется устойчивостью ее самого слабого звена). По мнению авторов, устойчивость системы в значительной степени зависит от того насколько данная система адаптирована к запросу надсистемы. Таким образом, устойчивая система $\mathbf{s}$ - это система, для которой: Os $\leftrightarrow \mathbf{f s}(\mathbf{L s}$ ?)Ls! $\leftrightarrow$ us, т.е. система, полностью адаптированная к запросу надсистемы. Степень устойчивости $\mathbf{s}$ определится степенью адаптированности/устойчивости $\mathbf{s}_{\mathbf{j}}$, которая представляет собой самое слабое звено, так $\mathrm{s}_{\mathrm{j}}$ не до конца адаптирована к функциональному запро- 
су со стороны $\mathbf{s}$. Данный закон работоспособен только при условии выполнения принципа самоорганизации, так как именно за счет механизма самоорганизации происходит адаптация элементов системы к их функциональным запросам. Учет данного принципа в процессе графоаналитического моделирования средствами УФО-подхода обеспечивается возможностью описания процесса адаптации системы к запросу надсистемы за счет изменения объектных характеристик.

Закон расхождения (различные части однородной системы подвержены действию сил, различающихся по качеству и величине, вследствие чего они изменяются различно) и ему противоположный закон опыта (единообразное воздействие на некоторое множество элементов уменьшает разнообразие состояний этого множества). Эти законы работоспособны только при условии выполнения принципа прогрессирующей механизации при разных (или одинаковых) функциональных запросах к разным системам. Учет данных законов в процессе графоаналитического моделирования средствами УФО-подхода обеспечивается возможностью описания процесса адаптации системы к запросу надсистемы за счет изменения объектных характеристик.

В стандарты системно-структурного, объектно-ориентированного графоаналитического моделирования и нотацию BPMN учет субстанциальных закономерностей не заложен.

\section{Выводы}

Исследованы возможности системно-объектного, системно-структурного (системно-функционального), объектно-ориентированного подходов и нотации BPMN учитывать общесистемные закономерности в графоаналитических моделях. Показано, что подход «Узел-Функция-Объект» $[15,16]$, позволяет это делать в большей степени, чем другие подходы.

\section{Благодарность: Работа выполнена при финансовой поддержке гранта РФФИ № 18-07-00356a.}

\section{Библиографический список}

1. Общая теория систем URL: https://ru.wikipedia.org/wiki/Общая_ теория_систем (20.04.2018).

2. Прангишвили И.В. Системный подход и общесистемные закономерности. М.: СИНТЕГ, 2000.

3. Маторин С.И., Зимовец О.А., Жихарев А.Г. Общесистемные принципы в терминах системно-объектного подхода «Узел-Функция-Объект» // Труды ИСА РАН. 2016. №1. Т. 66. С. 10-17.

4. Sergey I. Matorin, Aleksandr G. Zhikharev Calculation of The Function Objects as The Systems Formal Theory Basis // Advances in Intelligent Systems and Computing 679, pp. 182-191.

5. Abadi Martin and Luca Cardelli A Theory of Objects. SpringerVerlag. 1996. 
6. Zhikharev, A.G., Matorin, S.I., Zaitseva, N.O. About perspectives of simulation technological processes functioning with using systemobject approach node-function-object // International Journal of Applied Engineering Research, 10(12), pp. 31363-31370.

7. Мельников Г.П. Системология и языковые аспекты кибернетики. М.: Сов.радио, 1978.

8. Черемных С.В., Семёнов И.О., Ручкин В.С. Моделирование и анализ систем: IDEF-технологии: практикум. М.: Финансы и статистика, 2006.

9. Буч Г., Рамбо Д., Джекобсон А. Язык UML. Руководство пользователя / Пер. с англ. М.: ДМК, 2000.

10. Выдержки из перевода спецификации к нотации BPMN компании DIRECTUM [Электронный ресурc]. URL: http://www. DIRECTUM-Journal.ru/docs/1624827.html.

11. Matorin, S.I., Zimovets, O.A., Zhikharev, A.G. The elements of general theory of the systems in terms of system-object approach of «unit-function-object» // International Journal of Applied Engineering Research 10(24), pp. 44831-44837.

12. Zhikharev, A.G., Matorin, S.I., Kuprieva, I.A., Kulumbaev, E.B., Smirnova, S.B. The language of the description of the functional objects // Journal of Engineering and Applied Sciences, 12(Special issue 6), pp. 7859-7864.

13. Богданов А.А. Тектология: Всеобщая организационная наука. Сост., предисловие и комментарии Г.Д. Гловели, послесловие В.В. Попкова. М.: Финансы, 2003.

14. Маторин С.И., Соловьева Е.А. Детерминантная модель системы и системологический анализ принципов детерминизма и бесконечности мира // НТИ. Сер. 2. 1996. N8. С. 1-8.

15. Matorin, S.I., Zhikharev, A.G., Korchagina, K.V., Saitseva, T.V. About the development of new tools system-object simulation process and traffic //International Journal of Applied Engineering Research. 10 (24), pp. 45292-45296.

16. Zhikharev A.G., Matorin S.I., Zimovets O.A., Zhikhareva M.S., Rakov V.I. The simulation modeling of systems taking into account their internal parameters change // International Journal of Pharmacy \& Technology, vol. 8 (4), pp. 26933-26945.

\section{References}

1. Obshhaya teoriya sistem URL: https://ru.wikipedia.org/wiki/ Obshhaya_teoriya_sistem (20.04.2018) (General theory of systems. URL: https://ru.wikipedia.org/wiki/General_theory_systems (04/20/2018)).

2. Prangishvili I.V. Sistemnyj podkhod i obshhesistemnye zakonomernosti. M.: SINTEG, 2000 (Prangishvili I.V. System approach and system-wide regularities. Moscow: SYNTHEG, 2000)

3. Matorin S.I., Zimovets O.A., ZHikharev A.G. Obshhesistemnye printsipy $\vee$ terminakh sistemno-ob"ektnogo podkhoda «UzelFunktsiya-Ob"ekt» // Trudy ISA RAN. 2016. №1. Tom 66. S. 10-17 (Matorin SI, Zimovets OA, Zhikharev AG System-wide principles 
in terms of the system-object approach "Node-Function-Object" // Proceedings of the ISA RAS. 2016. №1. Volume 66. P. 10-17).

4. Sergey I. Matorin, Aleksandr G. Zhikharev Calculation of The Function Objects as The Systems Formal Theory Basis // Advances in Intelligent Systems and Computing 679, pp. 182-191.

5. Abadi Martin and Luca Cardelli A Theory of Objects. SpringerVerlag. 1996.

6. Zhikharev, A.G., Matorin, S. I., Zaitseva, N.O. About the perspectives of the simulation technology processes using the system-object approach node-function-object // International Journal of Applied Engineering Research, 10 (12), pp. 31363-31370

7. Mel'nikov G.P. Sistemologiya i yazykovye aspekty kibernetiki. M.: Sov.radio, 1978 (Melnikov G.P. Systemology and linguistic aspects of cybernetics. Moscow: Sov.radio, 1978)

8. CHeremnykh S.V., Semyonov I.O., Ruchkin V.S. Modelirovanie i analiz sistem: IDEF-tekhnologii: praktikum. M.: Finansy i statistika, 2006 (Cheremnykh SV, Semenov IO, Ruchkin VS Modeling and analysis of systems: IDEF-technologies: practical. Moscow: Finances and Statistics, 2006).

9. Buch G., Rambo D., Dzhekobson A. YAzyk UML. Rukovodstvo pol'zovatelya / Per. s angl. M.: DMK, 2000 (Buch G., Rambo D., Jacobson A. Language of the UML. User's Manual. with English. Moscow: DMK, 2000)

10. Vyderzhki iz perevoda spetsifikatsii $k$ notatsii BPMN kompanii DIRECTUM [EHlektronnyj resurs]. URL: http://www.DIRECTUMJournal.ru/docs/1624827.html (Extracts from the translation of the specification to BPMN notation from DIRECTUM [Electronic resource]. - URL: http://www.DIRECTUM-Journal.ru/docs/1624827. $\mathrm{html})$

11. Matorin, S.I., Zimovets, O.A., Zhikharev, A.G. The elements of the general theory of the systems in terms of the system-object approach of the "unit-function-object" // International Journal of Applied Engineering Research 10 (24), pp. 44831-44837

12. Zhikharev, A.G., Matorin, S.I., Kuprieva, I.A., Kulumbaev, E.B., Smirnova, S.B. The Journal of Engineering and Applied Sciences, 12 (Special issue 6), pp. 7859-7864

13. Bogdanov A.A. Tektologiya: Vseobshhaya organizatsionnaya nauka. Sost., predislovie i kommentarii G.D. Gloveli, posleslovie V.V. Popkova. M.: Finansy, 2003 (Bogdanov AA Tectology: General Organizational Science. Comp., Foreword and Commentary G.D. Gloveli, afterword of V.V. Popkov. Moscow: Finance, 2003)

14. Matorin S.I., Solov'eva E.A. Determinantnaya model' sistemy i sistemologicheskij analiz printsipov determinizma i beskonechnosti mira // NTI. Ser. 2. 1996. N8. S. 1-8 (Matorin SI, Solovyova EA The determinant model of the system and the systemological analysis of the principles of determinism and the infinity of the world // STI. Ser. 2. 1996. N8. Pp. 1-8)

15. Matorin, S.I., Zhikharev, A.G., Korchagina, K.V., Saitseva, T.V. About the development of new tools system-object simulation process and 
traffic // International Journal of Applied Engineering Research. 10 (24), pp. 45292-45296

16. Zhikharev A.G., Matorin S.I., Zimovets O.A., Zhikhareva M.S., Rakov V.I. The International Journal of Pharmacy \& Technology, vol. 8 (4), pp. 26933-26945

Рукопись поступила в редакцию: 26.07.2018, принята к публикации 26.08.2018

\section{6 авторах}

Маторин Сергей Игоревич, доктор технических наук, професcop, Researcher ID0, Scopus ID-6602150407, профрессор кафедры информационных систем и технологий Белгородского университета кооперации, экономики и права, Телефон: 8 (4722) 26-38-31, E-mail: matorin@bsu.edu.ru

Белов Сергей Павлович, доктор технических наук, профессор, Researcher ID A-6008-2014, Scopus ID - 56402764300, профрессор кафедры организации и технологии защиты информации Белгородского университета кооперации, экономики и права, Телесон: 8 (4722) 26-38-31, E-mail: belovssergei@gmail.com

Жихарев Александр Геннадьевич, кандидат технических наук, доцент, Researcher ID - L-6141-2016, Scopus ID - 56736375800, доцент кафедры информационных и робототехнических систем Белгородского государственного национального исследовательского университета. Телефон: 8 (4722) 30-13-76, E-mail: zhikharev@ bsu.edu.ru

\section{About author}

Matorin Sergey Igorevich, Doctor of Technical Sciences, Professor, Researcher ID - 0, Scopus ID - 6602150407, Chair of Information Systems and Technologies, Belgorod University of Cooperation, Economics and Law, Phone: 8 (4722) 26-38-31, E-mail: matorin@bsu.edu.ru

Belov Sergey Pavlovich, Doctor of Technical Sciences, Professor, Researcher ID A-6008-2014, Scopus ID - 56402764300, Chair of Organization and Information Security Technologies, Belgorod University of Cooperation, Economics and Law, Phone: 8 (4722) 26-38-31, Email: belovssergei@gmail.com

Zhikharev Alexander Gennadievich, Candidate of Technical Sciences, Associate Professor, Researcher ID - L-6141-2016, Scopus ID - 56736375800, Associate Professor of the Information Department and robotic systems of Belgorod State National Research University, Phone: 8 (4722) 30-13-76, E-mail: zhikharev@bsu.edu.ru 